\title{
Naming objects in BIM: A convention and a semi-automatic approach
}

\author{
Ke Chen ${ }^{1}$, Weisheng Lu ${ }^{2 *}$, Hongdi Wang ${ }^{3}$, Yuhan Niu ${ }^{4}$, and George G. Huang ${ }^{5}$
}

(1)

\section{Abstract}

A consistent and easily recognizable name is the primary identifier of an object in building information modeling (BIM). Existing naming conventions vary significantly from one to another, and require extensive manual work that is often tedious and error-prone. This study seeks for (a) developing a standardized naming convention for BIM objects, and (b) devising a semi-automatic naming approach for saving the manual work. In the proposed naming convention, each segment is included by referencing BIM standards and considering BIM users' actual needs; and the semi-automatic approach is formalized for both completed and ongoing BIM models. Validated by a control experiment and feedbacks from the project manager and BIM engineers of a real-life project, this research can be immediately applied to realize standardized BIM object names. This study also generates practical implications for BIM-based project management, where standardized BIM object names are required for supporting object identification and information incorporation throughout a project life cycle.

Keywords: Building information modeling; data interoperability; naming convention; Information technologies.

\section{Introduction}

\footnotetext{
${ }^{\text {a }} \mathrm{PhD}$ Candidate, Room533, Knowles Building, Department of Real Estate and Construction, The University of Hong Kong, Hong Kong, Email: leochen@connect.hku.hk;

b Associate Professor, Corresponding author, 5/F, Knowles Building, Department of Real Estate and Construction, The University of Hong Kong, Hong Kong, Email: wilsonlu@hku.hk;

c Postdoctoral Fellow, AC1-B7504, Department of Public Policy, City University of Hong Kong, Hong Kong, Email: wd.kevin@gmail.com;

${ }^{d}$ PhD Candidate, Room535A, Knowles Building, Department of Real Estate and Construction, The University of Hong Kong, Hong Kong, Email: yuhanniu@ connect.hku.hk;

e Professor, 8/F, Haking Wong Building, Department of Industrial and Manufacturing Systems Engineering, The University of Hong Kong, Hong Kong, Email: gqhuang@ @ku.hk
} 
Building information modeling (BIM) has revolutionized the way in which buildings are conceived, designed, constructed, and operated (Hardin and McCool 2015). In a BIM model, all objects (e.g., wall and window) are augmented with both geometric and non-geometric parameters (Pratt 2004). Among these parameters, the name is the primary identifier of a BIM object (Taylor 2007, Duddy et al. 2013, Chen et al. 2015), which is frequently used to link the object with relevant information in other data carriers such as Excel files or Word files throughout a project life cycle (Goedert and Meadati 2008). By doing so, project managers can truly use BIM to support process control (Song et al. 2012), facility management (Teicholz 2013), design assessment (Cidik et al. 2010), indoor navigation (Isikdag et al. 2013), safety checking (Zhang et al. 2013), energy simulation (Cao et al. 2014) and so on. Lacking standardized BIM object names would make it onerous for BIM users to retrieve information (Gandhi and Jupp 2014), and cause coordination difficulties and confusions amongst stakeholders (Taylor 2007, Shafiq et al. 2012).

In light of the importance of BIM object names, several naming conventions have been developed over the past years, though, not yet been widely used by practitioners. A primary barrier therein could be an insufficient analysis on the naming-convention segments regarding the practitioners' actual needs. Moreover, naming objects in BIM in a consistent and structured manner often involves extensive manual work that can be incredibly tedious and error-prone, particularly when construction projects nowadays become increasingly complex (Williams et al. 2014). This research thus aims to (1) develop a standardized naming convention for BIM objects that considers practitioners' requirements and meanwhile is largely compatible with prevailing BIM standards; and (2) devise a semi-automatic approach to naming BIM objects based on the proposed naming convention. 
46 This paper starts with a review of existing naming conventions and naming approaches. The processes of developing a viable naming convention and devising a semi-automatic naming approach are subsequently introduced. Next, validation of the naming approach is presented. Finally, concluding remarks are provided by highlighting implications of this paper and suggesting future research direction.

\section{Literature Review}

A structured BIM object naming convention is important for BIM users to easily understand and recognize denominated BIM objects (McPhee 2014, Barbosa et al. 2016). Existing BIM software such as Autodesk Revit, however, only labels BIM objects with default serial numbers or numerical identifications (IDs) that are meaningless to users. Facing this issue, some BIM standards (e.g., DOA/DSF 2009, National Building Specification [NBS] 2014) and scholarly papers (e.g., Pavan et al. 2014, Merschbrock and Munkvoid 2015) have suggested standardized naming conventions. These suggestions, nevertheless, have not provided sufficient details on the segments to be included in the names, and not attached adequate attentions to practical requirements of BIM users.

Previous studies have also striven to facilitate naming objects when developing the BIM model. For example, Eastman (2009) laid out a review tool for assessing whether objects in a model have proper names or not. Venugopal et al. (2012) suggested that rules should be written to check BIM object names. These efforts, unfortunately, only focused on ex post facto checking instead of ex ante assurance. Alternatively, some add-on tools enabling information exchange between BIM software and external files could be used to name BIM objects. For instance, BIMLink can export a list of BIM objects into an Excel file. Practitioners then can input a connotative name of each object in Excel, and import the file 
back to BIM. Such approach, though proving the importance of BIM object names, involves reiterative file exporting and importing, and still demands considerable manual work for searching and linking the information contained in BIM and its corresponding external file. Therefore, without an efficient and easily implemented naming approach, it would be difficult to assign standardized, connotative names to BIM objects, and in turn, to make a BIM model truly informative.

\section{Research Design and Methods}

This research was conducted via three steps (cf. Fig. 1). In the first step, the authors reviewed the existing naming conventions appeared in various BIM standards, and interviewed representatives of the Architecture, Engineering, and Construction (AEC) industry to collect their opinions on BIM object naming conventions. As a result of Step 1, a viable naming convention was developed. In the second step, each segment of the proposed naming convention was analyzed to identify whether it could be automatically acquired from BIM or needed to be input manually. The prerequisites for ensuring the quality of automatic acquisition were also analyzed. These analyses helped to devise the semi-automatic naming approach. In the third step, a control experiment was performed to quantitatively validate the devised semi-automatic naming approach. Besides, the proposed naming convention and the semi-automatic naming approach were introduced in a real-life project for analyzing their pros and cons.

$<<$ Please insert Fig. 1 here $>>$

\section{Towards a naming convention for BIM objects}

After screening 22 BIM standards published worldwide and written in English, the authors discovered that eight standards are particularly related to naming objects in BIM. They are 

a Semiautomatic Approach. Journal of Construction Engineering and Management. 06017001:1-6"

BIM Guidelines and Standards for Architects and Engineers (DOA/DSF 2009), E/A Design Division BIM Standard Manual (Port Authority of NY \& NJ Engineering Department [PANY\&NJED] 2012), Department of Design + Construction (DDC) - BIM Guidelines (DDC 2012), NBS BIM Object Standard (NBS 2014), Australian and New Zealand Revit Standards (Australian and New Zealand Revit Standard Committee [ANZRSC] 2012), BIMMechanical, Electric, Plumbing Australia Practice (Air Conditioning Mechanical Contractors Association [AMCA] 2014), AEC (UK) BIM Protocol (AEC UK 2012), BIM Library Components Reference (Hong Kong Housing Authority [HKHA] 2010). A total of ten naming-convention segments were regulated by the BIM standards reviewed, which are type (100\%), description (75\%), function (50\%), sequential number (50\%), manufacturer (50\%), location (37.5\%), role (12.5\%), item code (12.5\%), level of detail (12.5\%), and property $(12.5 \%)$. The percentage in brackets denotes how many percent of the standards have regulated that specific segment.

In order to gather opinions on name conventions from practitioners, the authors of this paper have interviewed 21 experts from 7 AEC firms in Hong Kong, including one private developer, one public developer, two leading main contractors, one design film, one principal supplier, and one global BIM software vendor. All interviewees possessed 3 years' or more experience of using BIM.

The interviewees were asked open-ended questions individually. Questions were organized into two parts, viz.,11.2 and Part II (P2) was to investigate their requirements for information segments to be included in a BIM object name. The $P 1$ data were transformed into weighting using principal component analysis (PCA) in SPSS. Given each interviewee's weighting and similar to the method used in the survey on BIM standards, the $P 2$ data were organized 
according to the weighted percentage assigned to each naming-convention segment mentioned by the interviewees. A total of nine segments were suggested. In descending order of the weighted percentage, they are type (100\%), location $(87.07 \%)$, sequential number (76.19\%), function (72.79\%), description (49.66\%), designer (14.29\%), manufacturer $(9.52 \%)$, item code $(6.80 \%)$, and level of detail $(4.76 \%)$. It means that all the interviewees opted in the importance of object 'type', $87 \%$ on 'location', and so on.

Segments that were agreed as being important were included into the proposed naming convention, while those with lower importance were excluded to avoid the redundancy of the BIM object names. The designer, project name, role and property were only mentioned in one of the two surveys and hence were not included. Although manufacturer was regulated by half of the BIM standards reviewed, the same was not true in the survey of AEC stakeholders (the view was that it would clutter up the BIM object name). The item code and level of detail were considered less important in both surveys, and so were dropped. As a result, five segments were incorporated in the proposed naming convention:

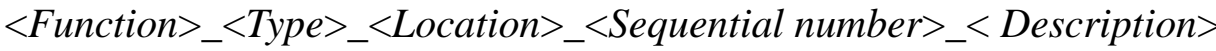

(1) Function gives the general classification of a BIM object (e.g., window, wall, or door).

(2) Type provides a detailed specification to distinguish objects having the same function (e.g., sliding door or pivot door).

(3) Location specifies where the object is located in the model. For objects that are not assigned to a specific level or space, this segment will be set to a null value.

(4) Sequential number refers to numbers in sequential order. It will be added when other segments in the names of any two or more objects share the same values.

(5) Description refers to supplementary information about the object. This may vary among projects and modelers, and thus could be considered as optional. 


\section{Developing the semi-automatic naming approach for BIM}

By applying the convention above, names of BIM objects become more meaningful and can serve as efficient identifiers. Nevertheless, to name the objects, including their detailed segments, is quite tedious, time-consuming, and prone to errors. Therefore, an automatic approach for naming BIM objects is desired. It has to be judged whether a segment can be

152 automatically acquired from BIM, or have to be input manually. By examining the mainstream BIM software, it is encouraging to discover that values of three segments of the proposed naming convention, viz., function, type, and location, can be obtained automatically when a BIM is developed, e.g., by retrieving BIM objects and putting them in place, if these values are preset in the software or added by BIM users. The sequential number can also be assigned automatically in an ascending numerical order in case of multiple identical objects (e.g., three windows) in one cluster (e.g., in a single wall). By contrast, description has to be manually added, edited, or deleted according to different requirements of different projects and stakeholders.

Based on the analyses, a semi-automatic naming approach is developed for both a completed and ongoing BIM (cf. Fig. 2). Here, 'completed' means that all information and details of the model have been set and no further changes are necessary; 'ongoing' means that the model is being developed and may change. For both, the first step is to specify the format of each naming-convention segment. The model should then be properly prepared to ensure that the name of each object contains the correct information in the correct format. The next step is to acquire the information for each segment by following a logical sequence. Finally, the collected information is combined to give the object name, and description will be manually added if required. When a BIM object is changed or moved in an ongoing BIM, its name 
should also be updated. Fig. 2 shows three scenarios for naming changed or moved objects.

In Case 1, the original object is replaced by a new one, and the entire naming process is repeated for this new object. In Case 2, the original object is moved, so the information of location is re-acquired for updating the location segment in its name. In Case 3, only description need to be added or removed manually with the other information remaining unchanged. All changes in objects' names need to be shared with all stakeholders in a timely manner to avoid misunderstandings or errors caused by inconsistent naming. The semiautomatic naming approach was programmed as an add-on tool for Autodesk Revit. This is called 'semi-automatic' since some of the segments of a name still need manual inputs. $<<$ Please insert Fig. 2 here >>

\section{Validation and discussion}

To validate the effectiveness of the proposed naming convention and the 'semi-automatic' add-on tool, a succession of research activities, primarily involving a control experiment in an education environment and feedback collection from the industry, were conducted.

\section{Control experiment}

The experiment was carried out in a university BIM lab that provided high-specification desktop computers with the same configurations and the BIM software Autodesk Revit 2015®. A BIM model of a high-rise public housing project was developed. The experimental task was to name the 191 prefabricated components of a typical floor in the BIM model. Specifically, experiment participants were requested to fill the 'Object Name' among the many properties of a component (cf. Fig. 3). 
A random sample of 32 Year-3 undergraduates participated in the experiment. They were all majored in construction and engineering. As most of them would probably work in the AEC industry in the near future, they were considered more similar to AEC practitioners than students taking other majors. The 32 participants were introduced to the subject project and the naming convention to be used (e.g., PF_TX8_2/F_2_M1, where 'PF' denotes for 'precast façade' [function], 'TX8' is the façade shape [type], '2/F' refers to 'the second floor' [vertical location], '2' refers to 'the second room' [horizontal location], and ' $\mathrm{M} 1$ ' is the mold type [description]), and were trained in basic operations of naming objects in Autodesk Revit $2015 \AA$. Then, the students were divided into two groups of equal size, and the experiment was conducted in four rounds. In the first two rounds, the control group was asked to manually input the name of each prefabricated component, as most of the existing practices do. In contrast, the experimental group was asked to use the add-on tool that was programmed to implement the semi-automatic approach (see Fig. 4). Then, the two groups switched tasks, and two further rounds of experiments were conducted. Therefore, each subject student could experience both manual input and the semi-automatic approach. $<<$ Please insert Fig. 4 here >>

The control group and the experimental group began tasks at the same time. Participants needed to alert the researchers once they finish their tasks on hand so the time they used is recorded and the accuracy rate was calculated instantly by dividing the number of correctly named objects by the total number of BIM objects. As shown in Table 1, by using the semiautomatic naming approach, the average time for completing the subject assignment could be shortened by $58.42 \%$, and the average accuracy rate can be increased by $9.36 \%$. This saving could be much more phenomenal in real-life BIM applications wherein a single floor could involve numerous objects. 


\section{Feedbacks from the industry}

224

By taking advantage of a government-funded research for applying BIM in a construction project, the proposed naming convention and the semi-automatic naming approach were introduced to the project manager and two BIM engineers. In the original BIM model of the subject project, all objects did not have standardized names. Considerable manual work hence was required for identifying individual BIM objects and matching them with the information in the enterprise information system (e.g., a logistic and supply chain management system) or other data carriers (e.g., a spreadsheet).

After specifying the format of each segment in the proposed naming convention and the BIM software used in this project, the add-on tool was provided to the three interviewees to implement the semi-automatic naming approach. Subsequently, they were invited to link BIM objects with their counterparts in an Excel file by using object names as identifiers. The usefulness of the semi-automatic naming approach was almost instantly confirmed by the interviewed BIM engineers. Besides, the project manager reflected that although extra time was spent for preparations such as checking the type of BIM objects, the efforts could be rewarded at later stages. Based on the proposed naming convention, an object name not only ensures quick, unique identification of a BIM object, but also facilitates information interoperability, which was key for conducting downstream analyses within BIM applications.

In addition to the favorable responses, interviewees expressed their concerns about the proposed naming convention. Firstly, it might be difficult for all stakeholders to follow the same naming convention without the leading role of the client or the main contractor. 
Secondly, the project manager expressed that the adoption of the naming convention would be affected by the extent to which BIM is truly implemented in a construction project. If the model was for 3D representation only, the naming convention would be less necessary. In contrast, to truly harness the power of BIM, succinct yet informative names of BIM objects become paramountly important.

\section{Concluding remarks}

As the most direct identifier of an object in BIM, a consistent and easy-to-recognize name is necessary for object identification and information interoperability. Without such name convention in place, it is almost suspicious to claim harvesting the full benefits of BIM in construction projects. This research, by developing a BIM object naming convention and encapsulating it in a computerized 'add-on' tool, has both immediate practicality and longterm significance. Firstly, a strategy is proposed for developing a naming convention that is compatible with prevailing BIM standards meanwhile meets practitioners' actual needs. By following this strategy, the naming convention, comprising five segments, can be easily interpreted by architects, engineers, contractors, and other stakeholders. Secondly, the devised semi-automatic naming approach can save BIM modelers tremendous amount of manual work when inputting the name of tens of thousands of objects in a BIM model. Future research could be undertaken towards enhancing the proposed naming approach by exploring translation of different depictions into a uniform one.

\section{Acknowledgements}

This study is jointly supported by the ITC Innovation and Technology Fund No. ITP/045/13LP, and the RGC General Research Fund No. 17205614. 


\section{References}

AEC UK. (2012). AEC (UK) BIM Protocol, London.

Air Conditioning Mechanical Contractors Association (AMCA). (2014). BIM-Mechanical, Electric, Plumbing Australia Practice, Victoria.

Australian and New Zealand Revit Standard Committee (ANZRSC). (2012). Australian and New Zealand Revit Standards, Australia.

Barbosa, M. J., Pauwels, P., Ferreira, V., and Mateus, L. (2016). “Towards increased BIM usage for existing building interventions.” Structural Survey, 34(2), 168-190.

Cao, J., Maile, T., O'Donnell, J., Wimmer, R., and van Treeck, C. (2014). "Model transformation from SimModel to Modelica for building energy performance simulation." Proc., $5^{\text {th }}$ German-Austrian IBPSA Conference, 242-249.

Chen, K., Lu, W. S., Peng, Y., Rowlinson, S., and Huang, G. Q. (2015). “Bridging BIM and building: From a literature review to an integrated conceptual framework." Int. J. Proj. Manage., 33(6), 1405-1416.

Cidik, M. S., Boyd, D., and Thurairajah, N. (2010). "Leveraging Collaboration through the use of Building Information Models.” Proc., 30 ${ }^{\text {th }}$ Annual ARCOM Conference, 713-722.

DDC (Department of Design + Construction). (2012). BIM guidelines, New York.

DOA/DSF (Department of Administration/Division of State Facilities). (2009). BIM guidelines and standards for architects and engineers, WI.

Duddy, K., Beazley, S., Drogemuller, R., and Kiegeland, J. (2013). "A platform-independent product library for BIM.” Proc., $30^{\text {th }}$ CIB W78 International Conference, 389-399.

Eastman, C. (2009). “Automated assessment of early concept designs.” Archit. Design, 79(2), $52-57$

Gandhi, S., and Jupp, J. (2014). "BIM and Australian Green Star Building 
Goedert, J. D., and Meadati, P. (2008). "Integrating construction process documentation into

Hardin, B., and McCool, D. (2015). BIM and construction management: proven tools, methods, and workflows. John Wiley \& Sons.

HKHA (Hong Kong Housing Authority). (2010). Building Information Modelling (BIM) Library Components Reference, Hong Kong.

Isikdag, U., Zlatanova, S., and Underwood, J. (2013). “A BIM-Oriented Model for supporting indoor navigation requirements." Comput. Environ. Urban, 41, 112-123.

McPhee, A. (2014). "The Nature of Naming. Practical BIM." <http://practicalbim.blogspot.hk/2014/08/the-nature-of-naming.html> (Oct. 1, 2016).

Merschbrock, C., and Munkvold, B. E. (2015). "Effective digital collaboration in the construction industry-A case study of BIM deployment in a hospital construction project." Comput. Ind., 73, 1-7.

NBS (National Building Specification). (2014). NBS BIM object standard. 〈https://www.nationalbimlibrary.com/nbs-bim-object-standard $\rangle$

Pavan, A., Daniotti, B., Re Cecconi, F., Maltese, S., Spagnolo, S., Caffi, V., Chiozzi, M., and Pasini, D. (2014). "INNOVance: Italian BIM Database for Construction Process Management." Computing in Civil and Building Engineering. 641-648.

PANY\&NJED (Port Authority of NY \& NJ Engineering Department). (2012). Engineering/Architecture Design Division BIM standard manual, New York.

Pratt, M. J. (2004). "Extension of ISO 10303, the STEP standard, for the exchange of procedural shape models.” Proc., Shape Modeling Applications, IEEE, 317-326.

Revit [Computer software]. Autodesk, San Rafael, CA.

Shafiq, M. T., Matthews, J., and Lockley, S. (2012). "Requirements for model server enabled 
collaborating on building information models." International Journal of 3-D Information Modeling, 1(4), 8-17.

Song, S., Yang, J., and Kim, N. (2012). "Development of a BIM-based structural framework optimization and simulation system for building construction." Comput. Ind, 63(9), 895912.

SPSS version 22.0 [Computer software]. IBM, Chicago.

Taylor, J. E. (2007). “Antecedents of successful three-dimensional computer-aided design implementation in design and construction networks." J. Constr. Eng. Manage, 133(12), 993-1002.

Teicholz, P. M. (2013). BIM for facility managers, John Wiley \& Sons, Hoboken, N.J.

Venugopal, M., Eastman, C. M., Sacks, R., and Teizer, J. (2012). "Semantics of model views for information exchanges using the industry foundation class schema." Adv. Eng. Inform, 26(2), 411-428.

Williams, G., Gheisari, M., and Irizarry, J. (2014). "Issues of Translating BIM for Mobile Augmented Reality (MAR) Environments." Proc., Construction Research Congress 2014, 100-109.

Zhang, S., Teizer, J., Perez, E., and McDonald, M. (2013). “Automated safety-in-design rulechecking for capital facility projects." Proc., 13th International Conference on Construction Applications of Virtual Reality, 40-46. 


\section{List of Figures}

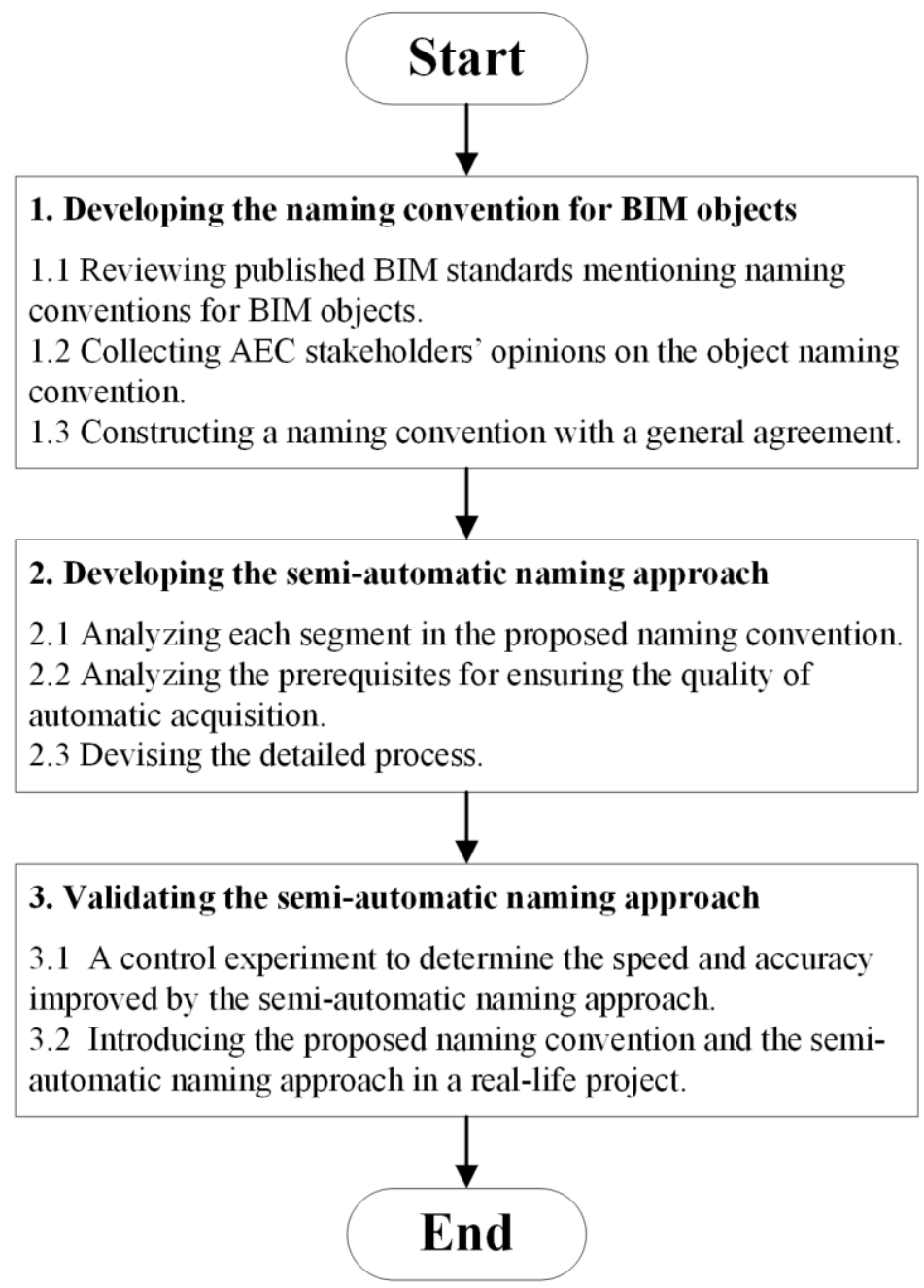

Fig. 1 Research methods 
Please cite this paper as "Chen K., Lu W., Wang H., Niu Y., Huang G.Q. (2017). Naming Objects in BIM: A Convention and a Semiautomatic Approach. Journal of Construction Engineering and Management. 06017001:1-6"

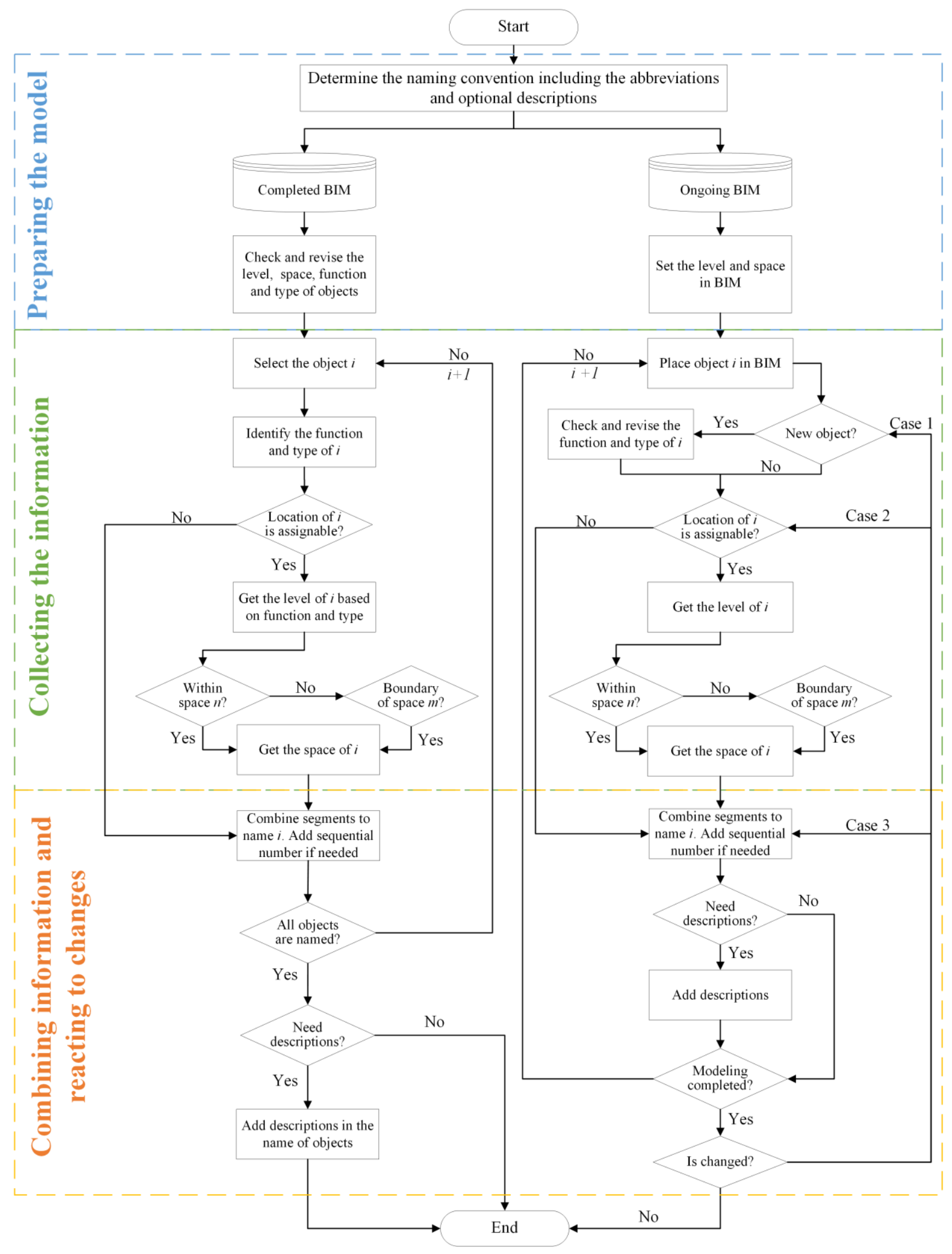

Fig. 2 The semi-automatic naming approach 


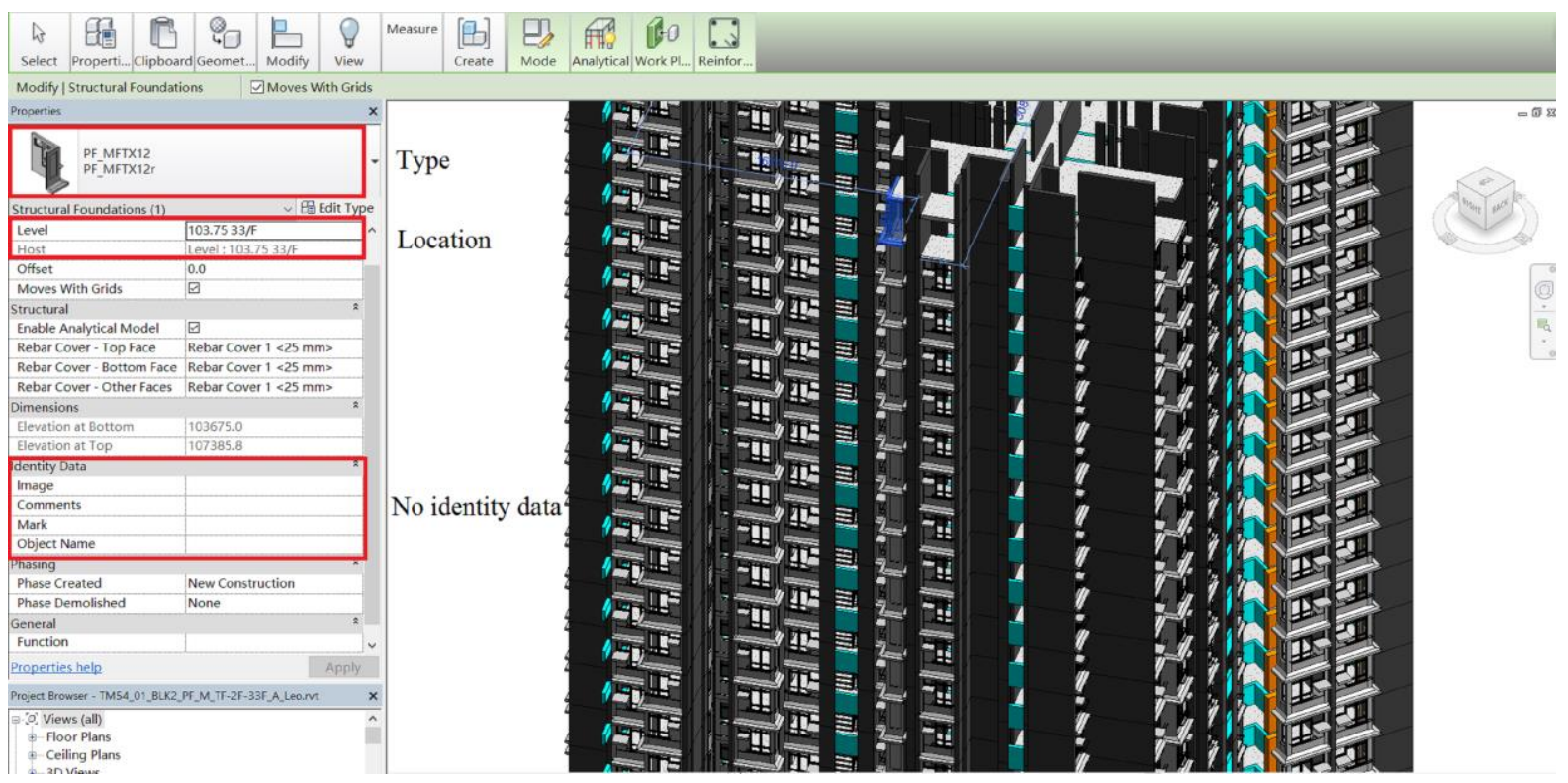

Fig. 3 The BIM model for the control experiment

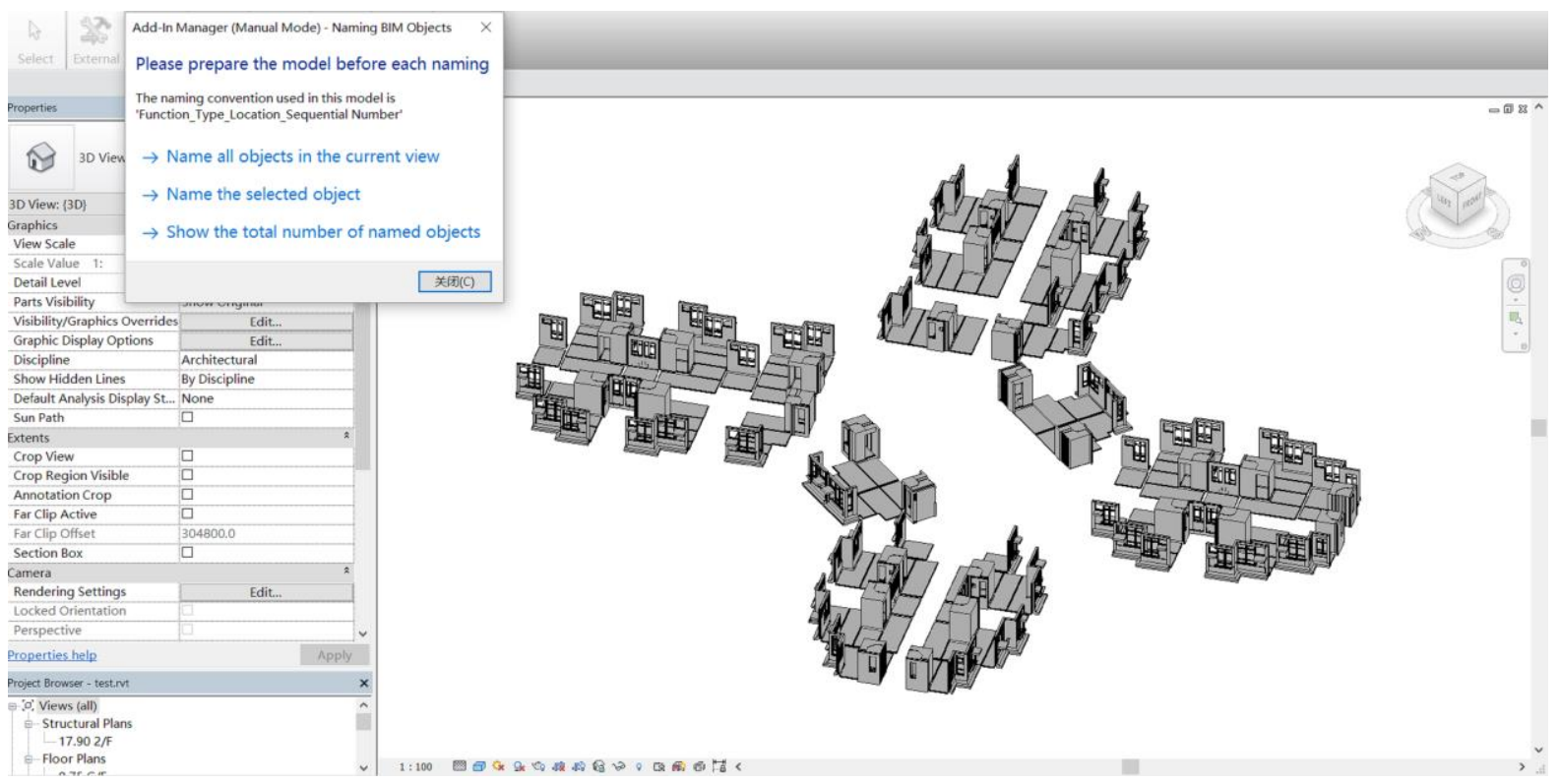

Fig. 4 Screenshot of the add-on tool 
Table 1. Results for time spent and accuracy rate.

\begin{tabular}{|l|l|l|l|l|l|l|l|l|}
\hline Group & \multicolumn{4}{|l|}{ Time spent (min) } & \multicolumn{3}{l|}{ Accuracy rate (\%) (Total = 191) } \\
\hline $\begin{array}{l}\text { Experimental } \\
(\mathbf{N = 1 6})\end{array}$ & Maximum & Minimum & Mean & SD & Maximum & Minimum & Mean & SD \\
\cline { 2 - 9 } & 37.79 & 28.76 & 33.27 & 2.9 & $\begin{array}{l}97.91 \\
(187)\end{array}$ & $\begin{array}{l}94.24 \\
(180)\end{array}$ & $\begin{array}{l}96.16 \\
(183.66)\end{array}$ & 2.22 \\
\hline $\begin{array}{l}\text { Control } \\
(\mathbf{N = 1 6 )}\end{array}$ & Maximum & Minimum & Mean & SD & Maximum & Minimum & Mean & SD \\
\cline { 2 - 9 } & 99.43 & 61.45 & 80.02 & 11.11 & $\begin{array}{l}91.10 \\
(174)\end{array}$ & $\begin{array}{l}83.77 \\
(160)\end{array}$ & $\begin{array}{l}87.16 \\
(166.47)\end{array}$ & 4.38 \\
\hline
\end{tabular}

